\title{
ESTUDO PALEOMAGNÉTICO DO VULCANISMO TERCIÁRIO DO ARQUIPÉLAGO DE FERNANDO DE NORONHA
}

\author{
ORLANDO TADEU LIMA DE SOUZA * e SONIA DIAS CAVALCANTE GUERREIRO*
}

\begin{abstract}
The Fernando de Noronha archipelago lies in the Atlantic Equatorial Ocean at $3^{\circ} 50^{\prime} \mathrm{S}$ latitude and $32^{\circ} 26^{\prime} \mathrm{W}$ longitude. It comprises a big volcanic complex consisting of unweathered volcanic rocks of Tertiary age. Three geological formations can be separated: Quixaba, Remédios, and São José. Using a compass and a clinometer, 100 samples were collected in the formations in a total of 15 sites. However, with the results obtained in the laboratory, it was impossible to make use of all studied sites because five out of the 15 sites presented weak magnetic intensity relative to the employed magnetometer preventing the cleaning process of the secondary magnetizations of these sites. Therefore, the 10 studied sites presented a magnetization with $\mathrm{D}=350^{\circ}, 1=-9^{\circ}, \alpha_{95}=6,8^{\circ}$, and $\mathrm{k}=51,2$. The cleaning process employed was the magnetic alternate field. Just one site prevented reversed polarization. The paleomagnetic pole was computed as $81^{\circ} \mathrm{N}$ and $232^{\circ} \mathrm{E}\left(\mathrm{A}_{95}=5^{\circ}\right.$, which agrees with the results for poles having the same age in the South America.
\end{abstract}

\section{SITUAÇÃO GEOLÓGICA E IDADE O Arquipélago} de Fernando de Noronha está localizado no Oceano Attântico equatorial, possui uma área de $18,4 \mathrm{~km}^{2}$ aproximada. mente e se encontra a $3^{\circ} 50^{\prime} \mathrm{S}$ de latitude e $32^{\circ} 26^{\prime} \mathrm{W}$ de longitude, no nordeste da costa brasileira.

Constitui-se de um grande edifício vulcânico não erodido do qual apenas uma pequena porção se acha emersa. A isóbata de $4.000 \mathrm{~m}$ na base da montanha é grosseiramente circular e apresenta um diâmetro de $60 \mathrm{~km}$.

A geologia do arquipélago foi estudada por Almeida (1958) e foram definidas três formações distintas.

A Formação Remédios é constituida por rochas eruptivas e instrusivas composta de substratos de rochas piroclásticas atravessadas por inúmeros diques e corpos intrusivos hipoabissais, traquitos e outras rochas de caráter ultrabásico-alcalino. As rochas desta formação são responsáveis pelas maiores elevações da área central da ilha principal do arquipelágo. As espessuras expostas na costa da Enseada da Caieira ultrapassam $100 \mathrm{~m}$ (Almeida 1958).

A Formação Quixaba é essencialmente composta por derrames de ankaratrito que recobrem as rochas erodidas da Formação Remédios. Sua espessura varia de alguns centímetros a cerca de $200 \mathrm{~m}$. As feições desta formação contrastam fortemente com as daquela subjacente por sua simplicidade, uniformidade e caráter extrusivo (Almeida 1958).

Estas duas formações ocorrem essencialmente na ilha principal do arquipelágo, porém, próximo a ela, assinalada por três illhotas, a de São José, Cuscuz e de Fora, e predominada por derrames de nefelina basanito distingue-se a Formação São José com espessura de, pelo menos, $25 \mathrm{~m}$ em alguns pontos.

Cordani (1970) determinou para a Formação Quixaba a idade em torno de $3 \mathrm{Ma}$, ou seja, final do Plioceno. Às formações São José e Remédios, do final do Mioceno e início do Plioceno, atribuiu idade de cerca de 9 Ma tendo utilizado o método K/Ar.

\section{AMOSTRAGEM E MEDIDAS PALEOMAGNETI-} CAS Utilizando-se uma perfuradora portátil foram coletadas 100 amostras de $2,5 \mathrm{~cm}$ de diâmetro por aproximadamente $4 \mathrm{~cm}$ de altura de um total de 15 sítios envolvendo as três formações do Arquipelágo de Fernando de Noronha (Fig. 1, Tab. 1).

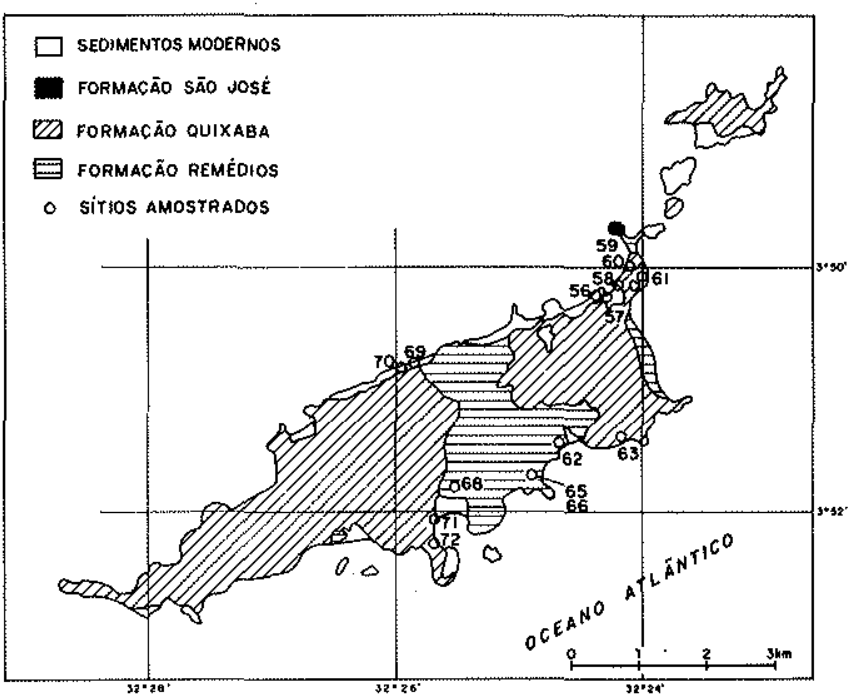

Figura 1 - Mapa esquemático mostrando os locais de amostragem no Arquipélago de Fernando de Noronha

As amostras foram orientadas no campo por meio de uma bússola magnética e clinômetro, e, a seguir, no laboratório, foram cortadas em cilindros de cerca de $2,4 \mathrm{~cm}$ de altura. Verificou-se a magnetização remanescente natural (MRN) de cada sítio utilizando um magnetômetro Foster. A lavagem das amostras foi feita através da variação de campo magnético alternado para se eliminar as possíveis magnetizaçōes secundárias, tendo sido utilizado nesta etapa um desmagnetizador Schostedt modelo GSD-1. A escolha do campo magnético ótimo para a lavagem foi feita com base no trabalho de Zjiderveld (1967) (Fig. 2). 
A determinação da magnetização remanescente característica (MRC) de cada sítio foi realizada de acordo com o trabalho de Fisher (1953), dando-se peso unitário à direção magnética apresentada por cada amostra. Da mesma forma, a magnetização remanescente característica da formação foi determinada dando-se peso unitário à direção magnética média de cada sítio. A média de todos os sítios estudados forneceu a direção (declinação e inclinação) do campo mag. nético da Terra à época que as rochas estudadas se formaram (3-9 Ma) - declinação do campo magnético em $350^{\circ}$ e inclinação em $-9^{\circ}$ (Fig. 3 ).

Foram determinados em seguida os pólos geomagnéti cos virtuais (PGV) de cada sítio (Fig. 4) para se chegar finalmente ao pólo paleomagnético que representa a média dos sítios do Arquipélago de Fernando de Noronha. Estes dados podem ser observados na tabela 1.

Não foi possível, com os resultados obtidos em laboratório, utilizar todos os sítios estudados, uma vez que cinco desses sítios apresentaram fraca intensidade magnética em relação ao magnetômetro utilizado, o que impossibilitou o processo de lavagem das magnetizaçốes secundárias dos mesmos (Tab. 1).

DISCUSSÃo DOS RESULTADOS Observando-se os resultados obtidos para as formações, de acordo com o exemplo da figura 3 , verifica-se que a lavagem magnética reduziu o espalhamento dentro dos sítios (Fig. 5). Este resultdado é coerente com os trabalhos de Richardson \& Watkins (1967) e Mazoires et al. (1971) para a ilha.

Os dados, quando avaliados conjuntamente para as formações, possibilitam a determinação de um pólo paleomagnético médio para o Arquipélago de Fernando de Noronha: $81^{\circ} \mathrm{N}$ de latitude e $232^{\circ} \mathrm{E}$ de longitude (com $\mathrm{A}_{95}=5^{\circ} \mathrm{e}$ $K=83$ ) (Tab. 1).

O resultado é compatível com o pólo paleomagnético encontrado por Richardson \& Watkins (1967) e Mazoires et al. (1971) no mesmo local ou, ainda, com o pólo terciário do Peru, determinado por Creer (1970); o pólo paleomagnético terciário da Argentina, determinado por Creer \& Valêncio (1969); e o pólo paleomagnético terciário da Itha Trindade, determinado por Valêncio \& Mendía (1974).

Não houve necessidade de serem feitas correções tectônicas, uma vez que a área não apresenta evidências de tectonismo expressivo após a formação das rochas estuda* das. Os sítios próximos entre si foram analisados estatisti. camente pelo teste $\mathrm{F}$ (que compara as direções médias dos vetores do campo magnético dos sítios estudados) (Watson 1956), para se verificar se os mesmos eram distintos uns dos outros ou se deveriam ser considerados como um único sítio. Os resultados indicaram que os sítios podem ser considerados, dentro de uma probabilidade de $95 \%$, como de sítios distintos, pois os valores encontrados para os testes se mantiveram acima do valor crítico indi-

Tabela 1 - Resultados paleomagnéticos das rochas vulcânicas do Arquipélago de Fernando de Noronha

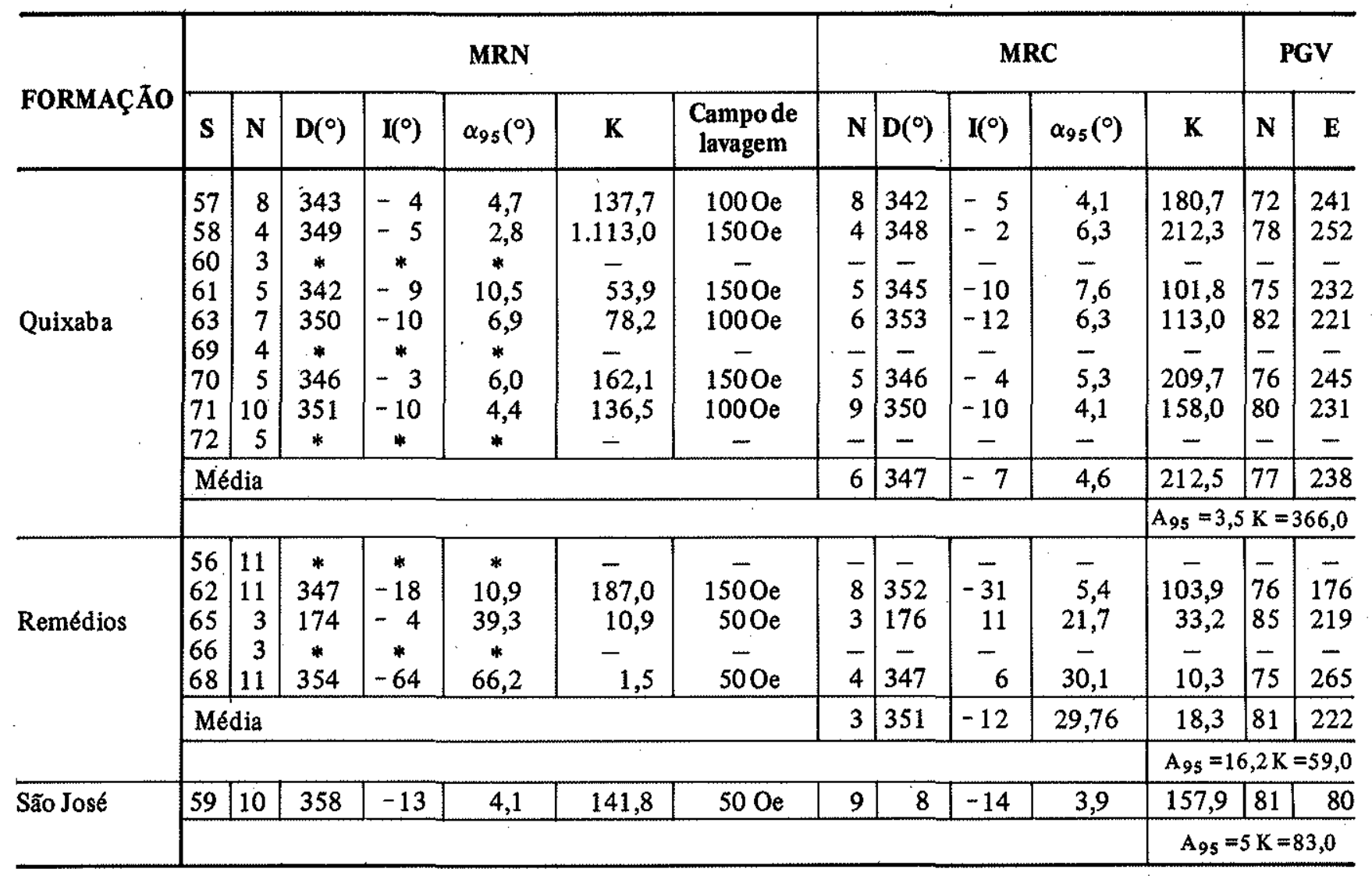

$\mathrm{N}=$ número de amostras; $\mathrm{S}=$ sítios; $\mathrm{D}=$ declinação; $\mathrm{I}=$ inclinação; $\alpha_{95}$ ou $\mathrm{A}_{95}=$ raio do círculo com $95 \%$ de confiança; $\mathrm{K}=$ parâmetro de precisão; $*$ não permitiu obter medidas. 

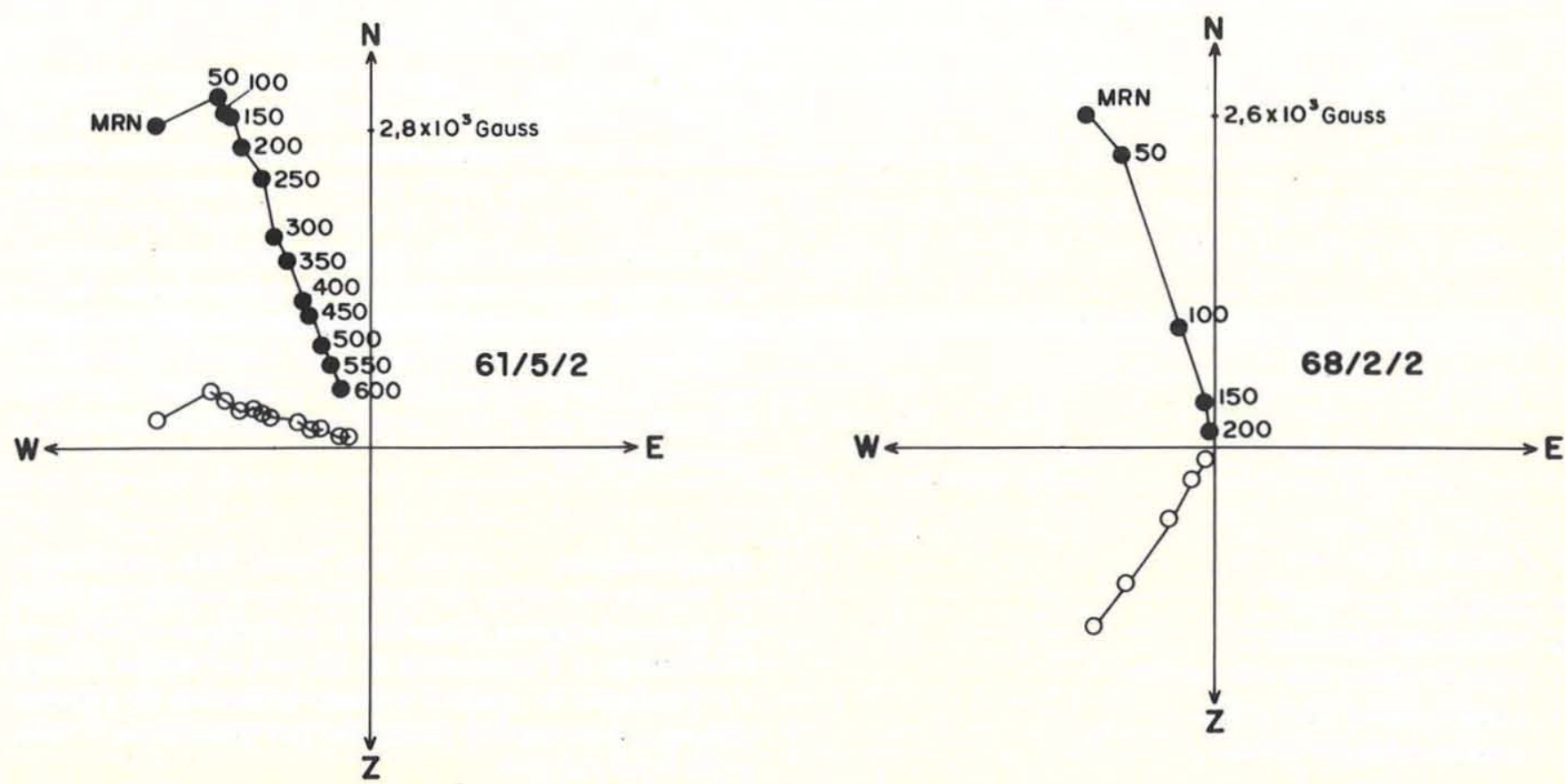

Figura 2 - Diagramas de Zjiderveld para as amostras 61/5/2 (Quixaba) e 68/2/2 (Remédios) do Arquipélago de Fernando de Noronha. Símbolos escuros e claros indicam, respectivamente, componentes no plano horizontal e vertical (EW)

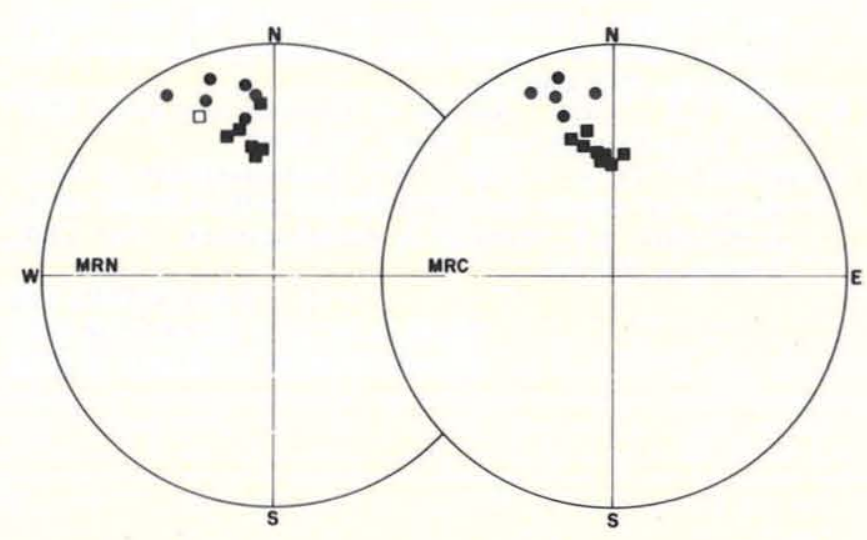

Sitio 61 (Ouixobo)

sitio 62 (Remédios)

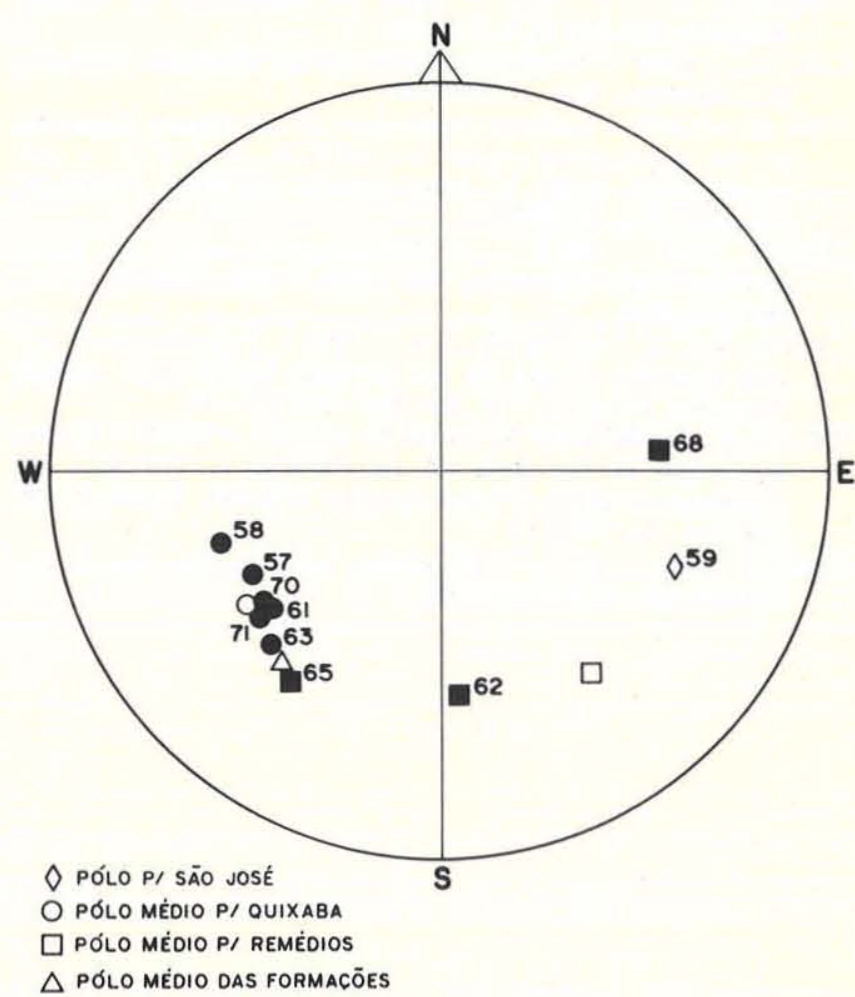

Figura 4 - Pólos geomagnéticos virtuais dos sitios do Arquipélago de Fernando de Noronha
Figura 3 - Direções médias do Arquipélago de Fernando de Noronha. Simbolos escuros e claros indicam, respectivamente, inclinação negativa e positiva. 


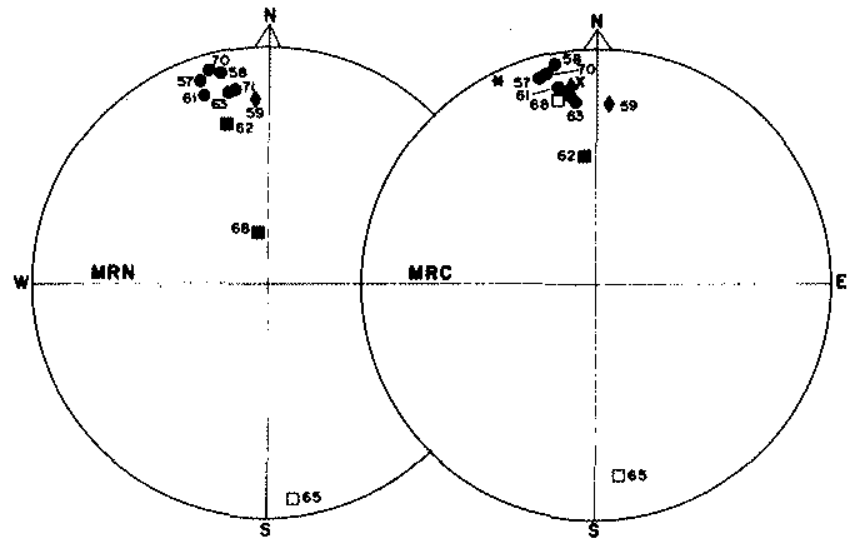

- formacäo sāo jösé

- formacão ouixaba

- formacão remédos

- oireçäo média das formacöes

* campo magnético atual

* campo dipolar atual

Figura 5 - Exemplos de redução do espalhamento dentro do sitio após o tratamento de desmagnetização. Símbolos escuros indicam inclinação negativa; claros, positiva. cado nas tabelas estatísticas, neste nivel de $95 \%$ de confiança.

$O$ desvio-padrão angular $\left(s=81 \mathrm{~K}^{-0,5}\right)$ foi calculado, a partir das direções médias dos sítios das formações, em $11,5^{\circ}$. A variação paleossecular encontrada (Cox 1969) apresenta valor um pouco abaixo das variações sugeridas pelos modelos A, B e D de Irving \& Ward (1964), Creer et al. (1959) e Cox (1970), respectivamente, que estudaram a dispersão angular das direções de magnetização. Entretanto, o fato de terem sido encontradas direções de magnetização normais e reversas sugere que a amostragem cobriu um tempo suficientemente longo, o que indica com boa aproximação, ser o pólo obtido um pólo verdadeiramente paleomagnético.

Agradecimentos Ao Dr. Axel Schult, pela contribuição no trabalho de coleta das amostras. Ao Conselho Nacional do Desenvolvimento Científico e Tecnológico ( $\mathrm{CNPq})$, ao Pró-Nuclear, à Financiadora de Estudos e Projetos (Finep) e à Fundação de Amparo e Desenvolvimento da Pesquisa (Fapesp), pelo suporte financeiro que possibilitou a realização deste trabalho.

\section{REFERENCIAS BIBLIOGRÁFICAS}

ALMEIDA, F.F.M. - 1958 - Geologia e Petrologia do Arquipélago de Fernando de Noronha. Rio de Janeiro, DNPM/DGM (Monografia 13).

CORDANI, U.G. - 1970 - Idade do vulcanismo do Oceano Atlântico Sul. Bol. IGA, 1 33-39.

COX, A. - 1969 - Confidence limits for the precision parameter K. J. Geophys. Res., 17:545-549.

COX. A. - 1971 - Latitude dependence of the angular dispersion of the geomagnetic field. In: BROCK, A. (1971) - An experimental study of palaeossecular variation. Geophys. $J, R$, astr. Soc., 24:303.317.

CREER, K.M. - 1970 - A Paleomagnetic survey of South American rock formations. Phil. Trans. R. Soc. London, Ser.A., 267:457-558.

CREER, K.M. \& VALENCIO, D.A. - 1969 - Palaeomagnetic and rock magnetic studies on the Cenozoic basalts from western Argentina. Geophys. J. R. astr. Soc., 19:113-146.

CREER, K.M.; IRVING, E.; NAIRN, A.E.M. - 1959 - Palaeomagnetism of the great sill. In: BROCK, A. (1971) - An experimental study of palaeossecular variation. Geophys. J.R. astr. Soc., 24:303-317.

FISHER, R.A. - 1953 - Dispersion on a sphere. Proc.R.Soc. London, Ser. A, 217:295-305.
IRVING, E. \& WARD, M.A. - 1964 - A statistical model of the geomacnetic field. In: BROCK, A. (1971) - An experimental study of palaeossecular variation. Geophys. J.R. astr. Soc., 24: 303-317.

MAZOIRES, J.; MENOR, E.A.; ROCHE, A.; SAUCIER, H.; SIAL, A.N. - 1971 - Étude paléomagnétic de laves de l'ile Fernando de Noronha. C.R.Acad.Sc.Paris., 273:551-554.

RICHARDSON, A. \& WATKINS, N.D. - 1967 - Palaeomagnetism of Atlantic Island: Fernando de Noronha. Nature, $215: 1470-1473$.

VALENCIO, D.A. \& MENDIA, J.E. - 1974 - Palaeomagnetism and $\mathrm{K} / \mathrm{Ar}$ ages of some igneous rocks of the Trindade complex and the Valado Formation, from Trindade island, Brazil. Rev. Bras. Geoc., 4(2):124-132.

WATSON, G.S. - 1956 - Analysis of dispersion on a sphere.Mon. Not.R.astr.Soc., Geophys. Suppl., 7:160-161.

ZJIDERVELD, J.D.A. - 1967 - A.C. desmagnetization of rocks: analysis of results. In: COLLINSON, D.M.; CREER, K.M.; RUNCORN, S.K. Methods in paleomagnetism. New York, Elsevier. p. 254-286.

MANUSCRITO 410

Recebido em 12 de dezembro de 1986 Revisão aceita em 22 de maio de 1987

O que a Ciência pode fazer pelo homem não é suficiente. Isto đeve se subordinar ao que a Ciência pode fazer para o homem. 